\title{
Radiation-Related Malignant Neoplasm
}

National Cancer Institute

\section{Source}

National Cancer Institute. Radiation-Related Malignant Neoplasm. NCI Thesaurus. Code C27209.

A malignant neoplasm arising in a site exposed to radiation therapy. 